\title{
Optimization of a Language for Data Mining
}

\author{
Rosa Meo \\ Università degli Studi di Torino - Dipartimento di Informatica \\ corso Svizzera 185 - 10149 - Torino - Italy \\ e-mail:meo@di.unito.it
}

\begin{abstract}
Constraint-based mining has attracted in recent years the interest of the data mining research community because it increases the relevance of the result set, reduces its volume and the amount of workload. However, constrained-based mining will be completely feasible only when efficient optimizers for mining languages will be available.

This paper is a first step towards the construction of optimizers for a constraint-based mining language. It provides the guidelines for the comparison of classes of statements by means of the relationships existing between their result sets. Furthermore it identifies as useful information to the optimization the presence of unique constraints and functional dependencies in the schema of the database. We show the practical implications of the discussed principles with a set of algorithms designed for a specific mining language. These algorithms use also a new designed index. called mining index that allows to reduce the portion of the database to be read in response to some classes of queries. In these cases the workload of the mining engine is greatly reduced or completely avoided in a significant subset of the cases.
\end{abstract}

\section{Categories and Subject Descriptors}

H.2.8 [Database Management]: Database Applications-data mining; H.2.3 [Languages]: [query languages]; H.2.4 [Systems]: [query processing]; D.3.4 [Processors]: Compilers, Optimization

\section{General Terms}

Languages, Theory, Algorithms

\section{Keywords}

Association rules, constraints, query optimization

\section{INTRODUCTION}

In data mining, especially if the volumes of the data are very large, there exist many data patterns that satisfy a

Permission to make digital or hard copies of all or part of this work for personal or classroom use is granted without fee provided that copies are not made or distributed for profit or commercial advantage and that copies bear this notice and the full citation on the first page. To copy otherwise, to republish, to post on servers or to redistribute to lists, requires prior specific permission and/or a fee.

SAC 2003, Melbourne, Florida, USA

Copyright 2003 ACM 1-58113-624-2/03/03 _..\$5.00. certain problem. If the discovered patterns do not seem to be perfectly tailored to the expectations of the user, he/she can further refine his/her query in order to restrict the result set in a meaningful way to the more interesting data patterns. However, the drawback is that the overall computational work might be prohibitive if each new request submitted by the user is processed by the system ex-novo because of the tremendous number of data patterns involved. On the contrary, it can be adopted with success if the query language and the data pattern extraction engine are able to work in an incremental fashion, in the sense that they compute on the fly the requested result on the basis of a previously generated result set or intermediate data set. In this way the overall mass of work is accordingly limited. A level-wise algorithm that tackles the related problem of the incremental maintenance of the rule base when the database is updated is presented in [3].

In the last years more interest has been focused on constraint-based mining $[4,6,12,13,15,14]$ for several reasons. Constrained mining helps to reduce the volume of the result set; it helps the user/analyst to specify better the typology of the desired result and therefore increases its relevance; it reduces the amount of computational work performed by the system, provided that constraints can be used to reduce the search-space of the patterns solving a certain problem $[12,13,14]$. Finally, constraintbased mining is at the basis of iterative, interactive mining $[6,8,12]$. In particular, in the context of inductive databases proposed by Mannila and Imielinski in [5], interactive, constrained mining occurs by means of the use of special-purpose query languages. These new promising approaches to mining will become really effective only when efficient optimizers for the mining languages will be available, i.e., if it will be possible to execute a query exploting the available information in the database, such as the constraints in the schema, the indices or the (intermediate) results of other queries, previously executed.

This paper is a first step towards the construction of optimizers of query languages for mining. It provides the guidelines for the comparison of classes of queries by means of the existing relationships between their result sets. As we will see each of these relationships (dominance, inclusion and equivalence) foresees a class of algorithms for the extraction of data mining patterns that presents a decreasing degree of coupling with the database: with dominance both the database and the result sets of some other queries must be read and processed; with inclusion only the result set must be read and processed, while finally with equivalence, only the result set must be read with no need of processing. These properties are particularly interesting 
when we want to speed-up the execution of a succession of several correlated queries and reduce the volume of data to be read in response to each of them, as in the case of constraint based, iterative mining. In this paper we identify the conditions (presence of keys and functionally dependendent attributes) under which a certain relationship can be implemented with an algorithm characterized by a lower degree of coupling with the database. In particular, the relationship of dominance between two queries (which occurs most frequently) can be implemented with an algorithm that requires only the input of the result set of some other queries and not the database. The presented guidelines are general and not restricted to a certain language. They are useful to identify the relationships between classes of queries under the cited conditions and provide the premises to reuse the result sets of previously executed queries.

In this paper we show that these guidelines are valid adopting them for a specific mining language, the MINE RULE operator $[10,11]$. Thus we show with a set of sample algorithms that an incremental approach for the extraction of association rules from a relational database is feasible. We show also the necessity and importance of storing intermediate results during query processing. We identify for these purposes a specific data structure, called mining index. In this paper, the design of the mining index is presented in such a way that the results of previous queries are exploited. We show that when a mining index has been created for query $Q$, and the system evaluates a query $Q$ ', if certain relationships exist between $Q$ and $Q$ ' the system will only use the mining index and the results of $\mathrm{Q}$. This reduces the number of $\mathrm{I} / \mathrm{O}$ operations on the source database and the overall computational effort. Finally, we show the use of mining indices and of constraints in database schema in some simple algorithms that address some typical cases of MINE RULE queries.

The paper is organized as follows: Section 2 presents the MINE RULE operator, Section 3 studies the relationships between two mining queries and applies its results to the MINE RULE language. Section 4 discusses the results and applies them in a set of new algorithms. Finally Section 5 draws the conclusions.

\section{MINE RULE SYNTAX}

We briefly present here the MINE RULE operator. For a complete description refer to $[10,11]$. The syntax of a MINE RULE query is the following:

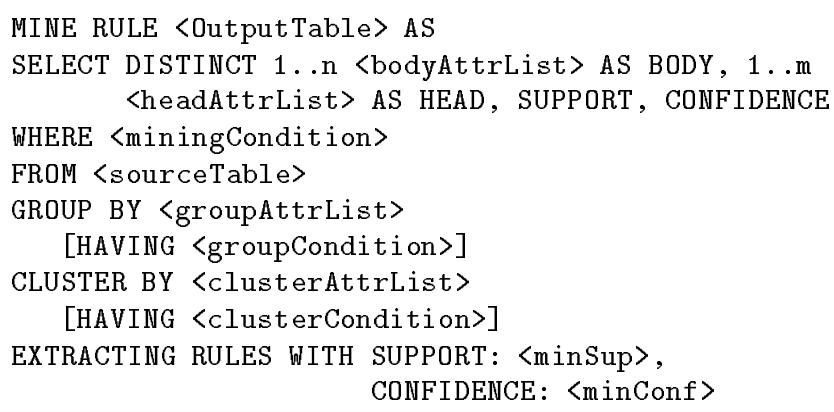

The association rules are extracted by performing the following steps:

Group computation. The GROUP BY clause logically partitions the source relation into groups, such that all tuples in a group have the same value of the grouping attributes groupAttrList.
Group filtering. The optional HAVING clause associated to the GROUP BY clause says that only groups in which all tuples satisfy the groupCondition are considered for rule extraction.

Cluster identification. The optional CLUSTER BY clause further partitions each group into sub-groups called clusters such that tuples in a cluster have the same value for the clustering attributes clusterAttrList. The body (respectively head) of a rule is extracted from clusters and not from entire groups. Thus elements in the body (respectively head) share the same value of the clustering attributes; if clusters are not specified, body and head are extracted from the trivial cluster, i.e. the entire group.

Cluster coupling. To compose rules, every pair of clusters (one for the body and one for the head) inside the same group is considered. Furthermore, the optional HAVING clause of the CLUSTER BY clause selects the cluster pairs that should be considered for extracting rules, that are those that satisfy the clusterCondition.

Rule extraction. From each group and each cluster pair, the SELECT clause extracts all possible associations of an unlimited set of bodyAttrList (clause $1 \ldots \mathrm{n}$ bodyAttrList AS BODY), representing the body of rules, with an unlimited set of headAttrList (clause 1 . n headAttrList AS HEAD), representing the head of rules. Attributes appearing in the rules are called rule attributes.

Mining condition. The (optional) wHERE clause following the SELECT clause forces rule extraction to consider only tuples that satisfy the miningCondition.

Support and confidence evaluation. The support of a rule is the number of groups from which the rule is extracted divided by the total number of groups generated by the GROUP BY clause. The confidence is the number of groups from which the rule is extracted divided by the number of groups that contain the body in some cluster. When support or confidence are lower than the respective minimum thresholds (minsup and minConf in our sample statement), the rule is discarded.

[11] pointed out that it is possible to write every MINE RULE query considering certain design criteria (which are the entities that association rules describe, how do we want to describe them, etc.). These criteria correspond to orthogonal dimensions in a cube, and in turn these dimensions correspond to the clauses of the MINE RULE statement: the GROUP BY and grouping attributes, the SELECT and rule attributes which are mandatory; the mining attributes (and relative predicates in mining condition), the clustering attributes (and relative predicates in clustering condition) which are optional ${ }^{1}$. For the purposes of the discussion we will refer to these attributes and conditions with simbols. The correspondences between attributes and simbols are indicated in Table 1.

${ }^{1}$ These latter considerations are of course valid also for the other query languages: for instance, in SQL the orthogonal dimensions are given by SELECT, WHERE, GROUP BY and ORDER BY clauses in which only SELECT is mandatory. 


\begin{tabular}{|c|c|}
\hline attributes or predicate & simbol \\
\hline grouping attributes & $\mathrm{ga}$ \\
grouping condition & $\mathrm{gc}$ \\
rule attributes & $\mathrm{ra}$ \\
mining condition & $\mathrm{mc}$ \\
clustering attributes & $\mathrm{ca}$ \\
clustering condition & $\mathrm{cc}$ \\
\hline
\end{tabular}

Table 1: Simbols for the main syntactic features of interest in MINE RULE

\section{THE RELATIONSHIPS BETWEEN TWO MINE RULE QUERIES}

As already said, the previous work on the containment relationships between two MINE RULE queries can be found in [1]. The main relationships between two queries are described in the following.

Equivalence Let $M$ and $M^{\prime}$ be two mining queries, extracting from the same source data rule sets $R$ and $R^{\prime}$ respectively. $M$ and $M^{\prime}$ are equivalent $\left(M \equiv M^{\prime}\right)$ if, for all instances of the source data, each rule $r$ in $R$ is also in $R^{\prime}$ and vice versa, with the same value of support and confidence.

Examples of equivalence are provided by the queries that are identical apart from the FROM clause in which two equivalent relations are retrieved (according to the relational algebra equivalence relationships). Other examples will be provided in presence of candidate keys or functional dependence relationships between attributes, and will be described in the paper.

Inclusion Let $M$ and $M^{\prime}$ be two mining queries, extracting from the same source data rule sets $R$ and $R^{\prime}$ respectively. $M$ includes $M^{\prime}\left(M \supseteq M^{\prime}\right)$ if, for all instances of the source data, each rule $r$ in $R^{\prime}$ is also in $R$ with the same value of support and confidence.

Examples of inclusion are provided by the queries $M$ and $M^{\prime}$ that are identical apart from the minimum support and confidence thresholds and the constraints on body and head minimum and maximum cardinalities [1].

Dominance Let $M$ and $M^{\prime}$ be two mining queries, extracting from the same source data rule sets $R$ and $R^{\prime}$ respectively, with $s, c$ and $s^{\prime}, c^{\prime}$ the support and confidence values of a rule in $R$ and $R^{\prime}$. Query $M$ dominates $M^{\prime}\left(M \triangleright M^{\prime}\right)$ if, for all instances of the source data, each rule $r$ in $R^{\prime}$ is also in $R$ with a support $s^{\prime} \leq s$ and a confidence $c^{\prime} \leq c$.

Examples of dominance are provided by the following theorem.

Theorem 1: Two queries, $M$ and $M^{\prime}$, identical apart from the respective mining conditions $m$ and $m^{\prime}$ are given. If $m^{\prime} \Longrightarrow m$ (i.e., $m^{\prime}$ entails $m$ ) then $M \triangleright M^{\prime} . M \triangleright M^{\prime}$ occurs also if the implication relationship $h^{\prime} \Longrightarrow h$ exists between the cluster conditions ( $h$ and $h^{\prime}$ ) of two mining queries $M$ and $M^{\prime}$, which are identical apart from the cluster condition predicates.

\subsubsection{Identifying the Dominant Queries}

We present here some general results that apply to MINE RULE queries. These results have been used also to elaborate the Sections that follow.
Theorem 2: Given queries $M$ and $M^{\prime}, M \triangleright M^{\prime}$ if $M^{\prime}$ is identical to $M$ apart from an additional clause. $\square$

As an example of the above Theorem, consider the following MINE RULE queries that extract association rules from the source table Purchases (tr, cust, item, date, price, discount) collecting purchase data of the customers transactions. In the example, query $M^{\prime}$ has an additional clustering clause.

\section{M: MINE RULE Output_for_query_M AS}

SELECT DISTINCT $1 \ldots$ item AS BODY,

$1 \ldots n$ item AS HEAD, SUPPORT, CONFIDENCE

WHERE BODY.price $>100$

FROM Purchases

GROUP BY cust

EXTRACTING RULES WITH SUPPORT:0.1, CONFIDENCE: 0.4

M': MINE RULE Output_for_query_M' AS

SELECT DISTINCT $1 \ldots \mathrm{n}$ item AS BODY, $1 \ldots n$ item AS HEAD, SUPPORT, CONFIDENCE

WHERE BODY.price $>100$

FROM Purchases

GROUP BY cust

CLUSTER BY date

EXTRACTING RULES WITH SUPPORT:0.1, CONFIDENCE: 0.4

The proof of the above theorem as well as of the theorems that will follow are omitted for lack of space but can be found in [2].

Theorem 3: Given a query $M$ and another query $M^{\prime}$ identical to $M$ apart from an additional predicate in gc, cc or $\mathrm{mc}: M \triangleright M^{\prime}$ if the additional predicate is connected with the conjunction operator; $M^{\prime} \triangleright M$ if it is connected with the disjunction operator.

\subsection{MINE RULE Queries and Candidate Keys}

In this Section we study if it is possible to derive the existence of relationships (equivalence, inclusion or dominance) between two MINE RULE queries by the presence of constraints defined in the database relation schema. In particular, we focus on the existing unique constraints defined over pairs of attributes, a and b, in the same table (or view). We suppose that this table or view is in the FROM clause of the MINE RULE queries. Furthermore, we suppose that each query refers by means of one of its clauses to at least one of them ( $a$ or $b$ ).

We start with some definitions and theorems. We will refer to them for the results that will follow.

Definition 1: We denote by a $\leftrightarrow$ b two candidate keys, i.e. two non null attributes of the same database relation on which a unique constraint is defined (unique (a) and unique (b)). In other words $\exists$ two functions $F, F^{-1} \mid \forall$ $\left(v_{a}, v_{b}\right), v_{a} \in \operatorname{Dom}_{a}$ (the domain of $a$ ), $v_{b} \in$ Dom $_{b}$ (the domain of $b)$, if $v_{b}=F\left(v_{a}\right)$ then $v_{a}=F^{-1}\left(v_{b}\right)$. $\square$

Theorem 4: Let be $\mathrm{a} \leftrightarrow \mathrm{b}$ and $M$ a query with $\mathrm{a} \in$ ga (or ca or ra). Let $M^{\prime}$ be a query identical to $M$ apart from ga (or ca or ra) in which $b$ is added to the attribute list (for instance, $\left.g a_{M^{\prime}}=g a_{M}, b\right)$. Under these conditions, $\mathrm{M}^{\prime} \equiv \mathrm{M}$. The same holds if $M^{\prime}$ is obtained from $M$ by substitution of $a$ with $b$.

Definition 2: Let be $\mathrm{a} \leftrightarrow \mathrm{b}$ and $\mathrm{P}(\mathrm{a})$ and $\mathrm{Q}(\mathrm{b})$ two predicates such that $\forall\left(v_{a}, v_{b}\right), v_{a} \in \operatorname{Dom}_{a}, v_{b} \in \operatorname{Dom}_{b}$, if $F\left(v_{a}\right)=$ 


\begin{tabular}{|c|c|c|c|c|c|c|c|}
\hline & \multicolumn{7}{|c|}{ MI } \\
\hline & & \multicolumn{3}{|c|}{ b } & \multicolumn{3}{|c|}{$Q(b)$} \\
\hline & & ga & $\mathrm{ca}$ & $\mathrm{ra}$ & $\mathrm{gc}$ & CC & $\mathrm{mc}$ \\
\hline M : & ga & $M^{\prime} \equiv M$ & $\mathrm{NA}$ & $\mathrm{NA}$ & NA & NA & $\mathrm{NA}$ \\
\hline \multirow[t]{2}{*}{ a } & ca & $\mathrm{NA}$ & $\mathrm{N}^{\prime} \equiv \mathrm{M}$ & $\mathrm{NA}$ & 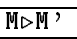 & M॰M & \\
\hline & ra & $\mathrm{NA}$ & $\mathrm{NA}$ & $\mathrm{N}^{\prime} \equiv \mathrm{M}$ & $\mathrm{NA}$ & NA & $\mathrm{NA}$ \\
\hline \multirow{3}{*}{$\begin{array}{l}\text { M: } \\
P(a)\end{array}$} & gc & $\overline{\mathrm{NA}}$ & $\bar{M}{ }^{\prime} \triangleright \mathrm{M}$ & $\mathrm{NA}$ & $\mathrm{M}^{\prime} \equiv \mathrm{M}$ & $\mathrm{M}^{\prime} \equiv \mathrm{M}$ & $\mathrm{N}^{\prime} \equiv \mathrm{M}$ \\
\hline & $\mathrm{CC}$ & $\mathrm{NA}$ & $\mathrm{H}^{\prime} \triangleright \mathrm{M}$ & $\mathrm{NA}$ & $\mathrm{H}^{\prime} \equiv \mathrm{M}$ & $\mathrm{H}^{\prime} \equiv \mathrm{M}$ & $\mathrm{H}^{\prime} \triangleright \mathrm{H}$ \\
\hline & $\mathrm{mc}$ & $\mathrm{NA}$ & & $\mathrm{NA}$ & $\mathrm{M}^{\prime} \equiv \mathrm{M}$ & $M \triangleright M$, & $\mathrm{M}^{\prime} \equiv \mathrm{M}$ \\
\hline
\end{tabular}

Table 2: The relationships between two MINE RULE queries in presence of candidate keys.

$\mathrm{v}_{\mathrm{b}}$ then $\mathrm{P}\left(\mathrm{v}_{\mathrm{a}}\right)=\mathrm{Q}\left(\mathrm{v}_{\mathrm{b}}\right)$. We call $\mathrm{P}(\mathrm{a})$ and $\mathrm{Q}(\mathrm{b})$ equivalent predicates. $\square$

Theorem 5: Let be $\mathrm{a} \leftrightarrow \mathrm{b}, \mathrm{P}(\mathrm{a})$ and $\mathrm{Q}(\mathrm{b})$ two equivalent predicates, and $M$ be a query with $\mathrm{P}(\mathrm{a})$ in $\mathrm{gc}$ (or cc or mc). Let $M^{\prime}$ be a query identical to $M$ apart from gc (or cc or $\mathrm{mc})$ in which $\mathrm{Q}(\mathrm{b})$ is added to the condition $\left(g c_{M^{\prime}}=\right.$ $\left.g c_{M} \wedge Q(b)\right)$. Under these conditions, $M^{\prime} \equiv$ M. The same holds if $M^{\prime}$ is obtained from $M$ by substitution of P (a) with $Q(b)$.

\subsubsection{Relationships between queries with reference to candidate keys}

In the analysis that follows, when we consider predicates in $\mathrm{gc}, \mathrm{m} c$ or $\mathrm{cc}$ of the MINE RULE queries we will refer to equivalent predicates, denoted by $\mathrm{P}(\mathrm{a})$ and $\mathrm{Q}(\mathrm{b})$. In Table 2 we report the relationships between two classes of MINE RULE queries when candidate keys are present. The two classes are identical apart from a single instantiated clause in each of them. Each of the instantiated clauses is indicated in one dimension of Table 2: the first query class, named $M$, is specified by the vertical dimension and its instantiated clause is defined with the assignment of a to the attribute list ga (or ca or ra) or with the predicate P (a) to the condition gc (or cc or $\mathrm{mc}$ ). The second query class, named $\mathrm{M}$ ', is specified by the horizontal dimension and its instantiated clause is defined with the assignment of $b$ to ga (or ca or ra) or with the assignment of $Q(b)$ to the condition gc (or $\mathrm{cc}$ or $\mathrm{mc}$ ). Furthermore, note that when $\mathrm{P}(\mathrm{a})$ is assigned to $\mathrm{gc}$ (or cc) it is an aggregate function expressed on the groups (clusters) and therefore it expects the grouping (clustering) is made by a. This means that $\mathrm{ga}=\mathrm{a}$ (or ca=a). The analogous reasoning holds for $\mathrm{P}(\mathrm{b})$ and $\mathrm{b}$.

To explain Table 2 we take as example the cell in the sixth row and second column (identified by the pair of assignments $\mathrm{m} c=\mathrm{P}(\mathrm{a})$ and $\mathrm{ca}=\mathrm{b}$ ) that is used to compare the following two classes of MINE RULE queries.

M: MINE RULE <0utputTable_for_M> AS

SELECT DISTINCT $1 \ldots \mathrm{n}$ 〈ruleAttr> AS BODY,

1..n <ruleAttr> AS HEAD, SUPPORT, CONFIDENCE WHERE $P(a)$

FROM 〈sourceTable

GROUP BY <groupAttr> [HAVING <groupCondition>]

EXTRACTING RULES WITH SUPPORT : $\langle\mathrm{mS}>$, CONFIDENCE: $:\langle\mathrm{mC}>$

M': MINE RULE <0utputTable_for_M'> AS

SELECT DISTINCT $1 \ldots \mathrm{n}$ 〈ruleAttr> AS BODY,

1..n <ruleAttr〉 AS HEAD, SUPPORT, CONFIDENCE

FROM 〈sourceTable〉

GROUP BY <groupAttr> [HAVING <groupCondition>]

CLUSTER BY $b$

EXTRACTING RULES WITH SUPPORT: $\langle\mathrm{mS}\rangle$, CONFIDENCE: $\langle\mathrm{mC}>$
Inside each cell of Table 2 we report the relationship between $\mathrm{M}$ and $\mathrm{M}$ '. When the cell is left empty nothing can be stated. If a cell contains NA it means that the comparison between the two classes of queries is Not Applicable because sufficient information is not available. In the case of the MINE RULE operator this is the case of the columns (rows) of ga and ra: if one of them is completely specified in a query class and not in the other class, it is impossible to compare the result sets of the two queries (unless supposed they are equal). Indeed, these two clauses (grouping and rule attribute selection) are the mandatory clauses without which a MINE RULE query is not meaningful.

With Table 2 we compare all the pairs of simplest, "basic" query classes and report their relationships. For the comparison of more complex queries, that exhibit a greater number of differing clauses you need to apply Theorems 2 and 3. This methodological way of comparing two classes of queries is valid for whichever query language and is not limited at all to the only MINE RULE operator. Indeed you need just to reserve one row/column of the table for each clause of the statement and a dimension of the table for each of the two query classes that you want to compare.

The detailed explanation of the content of Table 2 (and of the tables that follow) is omitted for lack of space; in [2] you can find the complete discussion. However it is important to observe that the equivalence relationship holds for every cell in the diagonal of Table 2 and this can be proved with the aid of Theorems 4 and 5 . Notice also that the table is symmetrical with respect to the diagonal. This is easy to understand for the symmetrical nature of the candidate keys and of the equivalent predicates.

\subsubsection{Determination of equivalent queries with ref- erence to candidate keys}

Now, we analyze the case in which the same query contains references to both candidate keys ( $a$ and $b$ ). In this case, we consider the classes of queries that are obtained by placing the two attributes in all the possible pairs of clauses. Table 3 shows all these pairs of clauses and therefore each cell of Table 3 identifies a single class of queries. For instance, the cell in the upper right corner corresponding to $\mathrm{ga}=\mathrm{a}$ and $\mathrm{m} \mathrm{C}=\mathrm{Q}(\mathrm{b})$, identifies the following class of MINE RULE queries

MINE RULE <0utputTable> AS

SELECT DISTINCT $1 \ldots$ n 〈ruleAttr〉 AS BODY, $1 \ldots \mathrm{n}\langle$ ruleAttr> AS HEAD, SUPPORT, CONFIDENCE WHERE $Q(b)$

FROM 〈sourceTable>

GROUP BY a [HAVING <groupCondition>]

CLUSTER BY <clustAttr> [HAVING <clustCondition>] EXTRACTING RULES WITH SUPPORT: $\langle\mathrm{mS}\rangle$, CONFIDENCE: $\langle\mathrm{mC}\rangle$

Inside each cell of Table 3 we specify the modifications to the query that allow us to obtain a new class of queries, equivalent to the previous class, i.e, such that their instances return the same result set, and this holds for every database. In particular, we specify in the row labelled out which of the two clauses can be omitted and in the row labelled in which new clause can be inserted in order to obtain the equivalent query class. If a cell is empty it means that nothing can be obtained for that combination of clauses. If a cell contains NA it means that the combinations of clauses is Not Applicable because it constitutes a meaningless combination of clauses. This is the case of $\mathrm{ga}_{\mathrm{a}} \mathrm{a}$ and $\mathrm{ra}=\mathrm{b}$ and vice versa ( $\mathrm{and}$ also $\mathrm{g} \mathrm{c}=\mathrm{a}$ and $\mathrm{ra}=\mathrm{b}$ and 


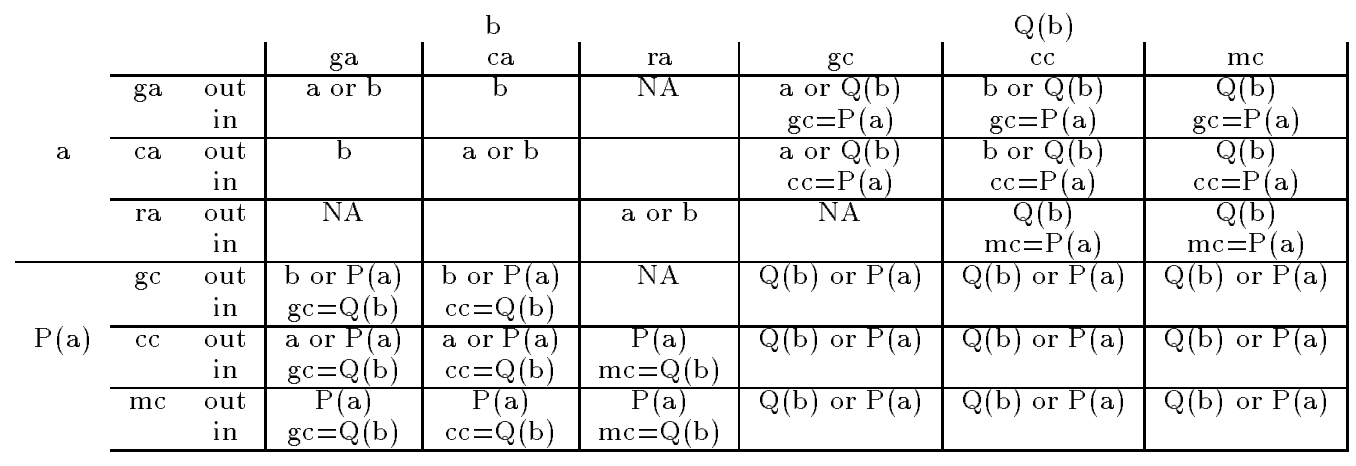

Table 3: Determining the equivalent MINE RULE queries in presence of candidate keys.

vice versa) in which only a single rule element can be extracted from each group and therefore no association rules result.

The query class in the above example is equivalent to the following query class where predicate $\mathrm{P}(\mathrm{a})$ is added to the grouping clause and mining condition $Q(b)$ is removed. Indeed, for all the tuples of each group (made by a) there is a unique value of $b$; as a consequence the mining condition $Q(b)$ is equivalent to the grouping condition $P(a)$.

MINE RULE <0utputTable> AS

SELECT DISTINCT $1 \ldots \mathrm{n}$ <ruleAttr> AS BODY, 1..n 〈ruleAttr〉 AS HEAD, SUPPORT, CONFIDENCE FROM 〈sourceTable〉

GROUP BY a HAVING <groupCondition> AND P (a)

CLUSTER BY <clustAttr> [HAVING <clustCondition>] EXTRACTING RULES WITH SUPPORT : $\langle\mathrm{mS}\rangle$, CONFIDENCE: $\langle\mathrm{mC}\rangle$

Notice that we have removed predicate $Q(b)$ from mining condition because our purpose is to identify the class of simplest queries (i.e. with the minimum number of predicates) that is equivalent to the original class.

This methodology is used in order to identify those classes of queries in which a certain degree of redundant information is present, where the redundancy is due to the presence of candidate keys in the query. Within the table we identify at least one class of equivalent queries, the simplest, by allowing a certain number of syntactical modifications to the initial query class. This allows us to obtain a simplified version of the same statement. Notice again that this methodology is valid for whichever query language and is not limited at all to the only MINE RULE operator.

In practice, the important result of the above conducted analysis consists in the identification of equivalent queries that constitute one of the major steps towards the construction of powerful query optimizers. Indeed, with equivalent queries the system does not need to perform a preliminary and heavy scan over the source data or processing the rule set but it needs just to retrieve the equivalent result set that is already present in the database. Furthermore, the above discussed tables identify query classes with a dominance relationship. In these cases, the algorithms with an incremental approach are possible and save some computation with respect to the non incremental ones. In Section 4 some of these algorithms are compared and discussed.

\subsection{MINE RULE Queries and Functional Dependence}

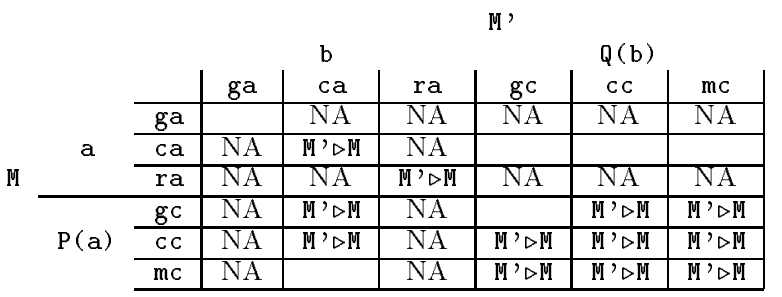

Table 4: The relationships between two MINE RULE queries in presence of functional dependence.

In this Section we study the relationships between two MINE RULE queries when a functional dependence exists between two attributes, $\mathrm{a}$ and $\mathrm{b}$ that belong to the same table (or view) in the FROM clause. Furthermore, we suppose that each query refers by means of one of its clauses to at least one of them ( $a$ or $b$ ).

Definition 3: We denote by $\mathrm{a} \stackrel{\mathrm{FD}}{\rightarrow} \mathrm{b}$ a functional dependence between $\mathrm{a}$ and $\mathrm{b}$. In other words, we mean that there exists a function $F$ such that given a value $v_{a} \in D_{0} m_{a}$ there exists a single value $\mathrm{v}_{\mathrm{b}} \in \operatorname{Dom}_{\mathrm{b}}$ such that $\mathrm{F}\left(\mathrm{v}_{\mathrm{a}}\right)=\mathrm{v}_{\mathrm{b}} . \quad \square$

Definition 4: Let be a $\stackrel{\text { FD }}{\rightarrow}$ b. We call $P(a)$ and $Q(b)$ functional preserving predicates if for each pair of values $\left(v_{a} \in\right.$ Dom $\left._{a}, v_{b} \in D m_{b}\right)$ such that $F\left(v_{a}\right)=v_{b}$ then $P\left(v_{a}\right) \Longrightarrow$ $\mathrm{Q}\left(\mathrm{v}_{\mathrm{b}}\right) . \square$

In the analysis that follows, when we consider predicates in $\mathrm{gc}, \mathrm{mc}$ or $\mathrm{cc}$ of the MINE RULE queries we will refer to functional preserving predicates, denoted by $\mathrm{P}(\mathrm{a})$ and $\mathrm{Q}(\mathrm{b})$. In Table 4 we report the cases in which the functional dependencies can be exploited for the purposes of the optimization and incremental execution of the query. In this case, we start our analysis by considering two classes of queries. Each class is identified by a single instantiated clause shown in one of the dimensions of Table 4. Inside each cell of Table 4 we report the existing relationship between the two classes of queries ( $M$ and $M^{\prime}$ ). This table is the analogous of Table 2 but designed for functionally dependendent attributes and functional preserving predicates. Notice that Table 4 is not symmetrical as the analogous Table 2 for candidate keys. This can be explained because functionally dependent attributes and functional preserving predicates cannot be substituted one with each other without changing the semantics of the query.

In the following we will not discuss in detail the content of the cells of Table 4 that can be easily derived with anal- 
ogous reasoning of Table 2. However, you can refer to [2] for a complete discussion.

Now, we analyze the case in which the same query contains both attributes related by a functional dependence (Table 5). In this case, we identify classes of queries that are obtained by placing the two attributes in all the possible pairs of clauses. Table 5 is analogous to Table 3 , but designed for functionally dependent attributes and functional preserving predicates. For lack of space we will not discuss in detail the content of Table 5 which can be easily derived applying analogous reasoning of Table 3. However, you can refer to [2] for a complete discussion.

The results contained in the Tables $2-5$, provide us important information that allow to determine precisely which queries are equivalent or with a dominance relationship. In the next Sections we will discuss in detail the classes of algorithms that can be adopted for these classes of queries. Furthermore, they allow us to observe that the cases of inclusion relationships are not frequent. These results are the premise that allow us to start with the construction of a MINE RULE optimizer and more in general to afford the optimization of mining queries with constraints.

\section{THE ALGORITHMS FOR SOME TYP- ICAL CASES}

The problem of association rules extraction typically requires tasks that are computationally expensive; therefore it is important to reduce them whenever possible. The incremental computation of the result set of a query $M^{\prime}$ starting from the result set of a previously executed query $M$ can provide significant reduction of the computational work perfomed by the system. In the general case, the problem is difficult to solve for several reasons. One of the reasons is that the association rules extraction is executed by a set of highly optimized procedures. Another reason is that pattern extraction in knowledge discovery is a task that performs aggregation of data. Under these conditions it is difficult to restore the original values from the aggregated ones, unless suitable intermediate information is maintained. This occurs in the data warehouse framework in which summarized data are maintained so that the computation of the aggregated data can be performed efficiently and at the same time, original data can be restored from them. This occurs also in the database mining framework in which given an itemset with an aggregated value (its support value) we need to efficiently retrieve all the rows of the original relation in which the itemset is present. This problem occurs typically during the incremental evaluation of a query $M^{\prime}$ from the result set of a previously executed query $M$ : the itemset whose rows have to be retrieved belongs to the result set of $M$, and the user-defined constraint on the attributes of those rows (such as the mining condition in MINE RULE) belongs to $M^{\prime}$. This issue will be discussed in detail in the following section on the intermediate data structure design.

However, in some particular case, incremental computation of the association rules is possible and decideable with ease. This occurs when certain relationships hold between the queries and certain conditions that we will discuss in the following are met. In particular,

Equivalence: if $M \equiv M^{\prime}$ then no computation is required because $R=R^{\prime}$.
Inclusion: if $M \supseteq M^{\prime}$ then $R^{\prime}$ can be obtained by $R$ without scanning the source data, but only with a scan of the rules in $R$ in order to select those rules that are also in $R^{\prime}$. Recall that this scan of the rule set $R$ is sufficient because $M \supseteq M^{\prime}$ occurs when the changed requirements of $M^{\prime}$ w.r.t. $M$ (like cardinality or support) can be checked directly in the relation containing the result set $R$.

Dominance: if $M \triangleright M^{\prime}$ then $R^{\prime}$ can be obtained by $R$ with a scan of $R$ and a single scan of the source data, needed in order to derive the correct values of support and confidence of the rules in $R^{\prime}$.

In $[1,2]$ the schema of an incremental computation for the case of dominance between two queries is reported. The algorithm considers each rule of $R$ as a candidate rule for $R^{\prime}$ and recomputes the rule support for all the groups in which the rule can be found. If the resulting support or confidence of the rule are not sufficient the rule is not included in the output rule set $R^{\prime}$.

This algorithm presents the problem that it performs a full scan of the database with the only purpose to computing the support and confidence values of a given set of rules. In theory, it would be sufficient to read only the portion of the database in which the given rules are present. This would be possible only if we were able to provide the system with more sophisticated data structures. This is the issue discussed in the following section. Furthermore, in Section 4.2 , we present an enhanced algorithm that makes use of this data structure.

\subsection{Design of Intermediate Data Structures: the Mining Indices}

In this Section we describe the intermediate data structures that are used by more general incremental algorithms in order to retrieve from the database only those data that are effectively needed. We designed these data structures with the following idea in mind. In traditional databases, an index is used by the system to make efficient access to a tuple searched by the value of some of its attributes. A similar need is present in the case of association rule mining. Given an itemset $I$, from which a rule $r$ in $R$ can be generated, we need to retrieve all the groups in which $I$ is present. This is because we want to evaluate the constraints of the new query $M^{\prime}$ on the rule $r$ in order to compute its support.

In theory, each itemset could have an entry in this data structure that we call mining index. In practice, this is not needed. Recall that the theory on anti-monotone and succinct constraints $[12,13]$ suggests that if a constraint is both anti-monotone and succinct at the same time (as most of the constraints in MINE RULE), it is sufficient to check it only for the first level of the lattice (i.e., only for the single items). Then, the same constraints are by sure also satisfied by all the itemsets that will be derived by combining the items at the first step. In this way, the constraints are effectively used to efficiently prune the lattice of the itemsets.

In conclusion, a mining index is designed as follows. We maintain an efficient and ordered access to the items (for instance by means of a hash table or a tree such as $\mathrm{B}+$ tree); for each entry we have all the identifiers of the groups in which that item is present. We indicate this data structure $I_{\text {item }}$ if the index is created on the item attribute. We will see that also other indices, on some other attribute list, 


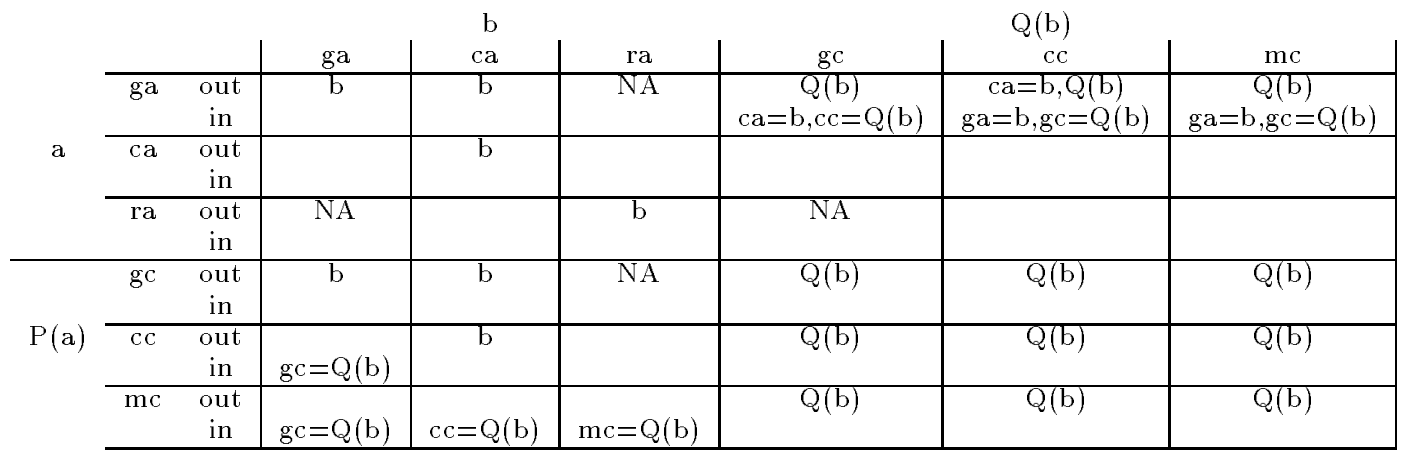

Table 5: Determining the equivalent MINE RULE queries in presence of functional dependence.

will be useful. For instance, if a MINE RULE query with a mining condition on attribute price (such as BODY.price $>100$ ) is submitted, the system can conveniently use also the mining index $I_{\text {price }}$ on the mining attribute. This index keeps the values of the price attribute ordered and for each value it gives the list of group identifiers in which there is a tuple with that value of price. In this way it is relatively easy to check if a rule in a previous result set still satisfies the constraints of the new query. This issue will be discussed in detail in the following Section.

\subsection{A New Enhanced Incremental Algorithm}

With the above observations, we propose a new enhanced incremental algorithm that works as follows. For the purposes of the description, to be concrete, we make use of a practical example. Suppose that a MINE RULE query $M$ with the mining condition B0DY.price $<150$ has been already executed. After some time, another query is submitted, $M^{\prime}$, that is identical to $M$ apart from a tighter mining condition (BODY.price $<100$ ). You can see that $M \triangleright M^{\prime}$ because between the mining conditions of $M$ and $M^{\prime}$ there is an implication relationship.

The intuition tells us that it is not necessary to read all the database in order to select from $R$ those rules that are valid also in $R^{\prime}$. It suffices to read only the groups in which at least a rule of $R$ is present. This in order to evaluate the constraints of the query $M^{\prime}$ on the tuples of the original relation. But perhaps we can do better and exploit the constraints that are present in this particular query. Actually, it might be necessary to only read the groups that contain both a rule in $R$ and a tuple with price that gets a value that satisfies the query constraint (price $<100$ that we denote with $\left.0 \mathrm{~K}_{\text {prices }}\right)$. However, if the cardinality of the complementary domain $(100 \leq$ price $<150$ denoted with $\mathrm{NO}_{\text {prices }}$ ) is lower a destructive approach is used and the new result set $R^{\prime}$ is obtained by elimination of a selected subset of rules from $R$. In the following (Figure 1), we give the algorithm with the constructive approach and show the use of the mining index.

The algorithm takes as input the result set $R$ of $M$ and gives in output the result set of $M^{\prime}$ : at first, searching in $I_{\text {price }}$ it builds the gid-list $\mathrm{g}-1$ ist-p containing the group id of groups with at least a tuple with a valid price. Then, for each rule $r$ in $R$, obtain $g$-list-temp with the group id of groups satisfying both $r$ and a valid price. Then the gidlist $\mathrm{g}-\mathrm{l}$ ist $-\mathrm{db}$ containing the id of the groups to be read from the database is formed. During the reading phase of the database only this portion of the database is scanned and from it the rules satisfying $M^{\prime}$ are generated (in $R S$ ).
Among these rules, only those ones that are already in $R$ are inserted in $R^{\prime}$ and kept if their support and confidence are sufficient (these ones can be determined by the gid-list associated to the rule).

This algorithm is more efficient than the original one in [1] whose complexity is $0(\mathbb{N})$ (with $\mathbb{N}$ the dimension of the database, see [16]), while the discussed algorithm has complexity $\mathrm{O}(\mathrm{N} * \mathrm{f})$ where $\mathrm{f}$ is the selectivity of the constraints in $M^{\prime}$ that are evaluated when searching groups in the mining index.

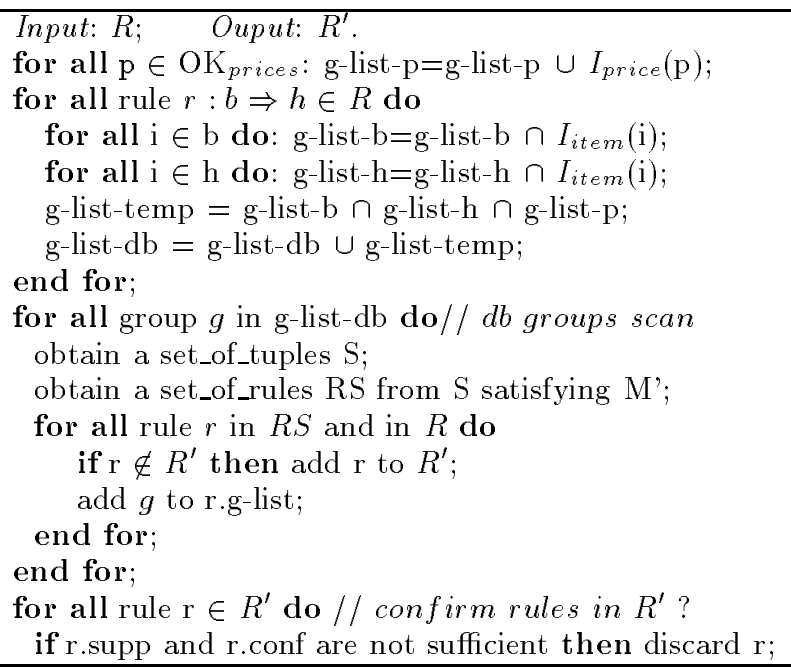

Figure 1: The generation of $R^{\prime}$ from $R$ with the use of the mining indices $I_{\text {item }}$ and $I_{\text {price }}$.

\subsection{The Functional Dependence between Rule Attributes and Mining Attributes}

In this section we describe a special case in which a functional dependence exists between the rule attributes and the mining attributes. We make reference to the same example discussed in the previous section in which the rule attribute is item and the mining attribute is price. Suppose there is a functional dependence item $\stackrel{\mathrm{FD}}{\rightarrow}$ price. When functional dependencies are present the system can keep a look-up table with the correspondencies between the attribute values. Let us call this table FD (item, price). In presence of functional dependencies the algorithm that we have described in the previous section is significantly more efficient because it can completely avoid the scan of the 
database in favour of a scan of the FD table (whose cardinality is far lower than the cardinality of the database since $\mathrm{FD}=\prod_{\text {item,price }}($ database $\left.)\right)$.

We report in Figure 2 the sketch of the algorithm. In general, there may be two subsets (possibly disjoint) of the values of the price domain that are allowed by the query $M^{\prime}$ : one for the body elements (denoted by $0 \mathrm{~K}_{\mathrm{B}-\mathrm{prices}}$ ), and another for the head elements $\left(0 \mathrm{~K}_{\mathrm{H} \text { prices }}\right)$. For each value of price in these two sets we obtain the set of values of item that are allowed in the body or in the head of the rules (resp. $i-l i s t-b$ and $i-l i s t-h$ ). This is an immediate operation that is performed by looking in the table FD(item,price). Then the algorithm performs one scan over $R$ and copies in $R^{\prime}$ the rules whose items are found in the previously identified lists. Notice that in this case the algorithm performs only a scan of the rule set $R$ and completely avoids the scan of the database. In other words, the algorithm is transformed from a "dominance" algorithm into a (more efficient) "inclusion" algorithm that is characterized by a lower degree of coupling with the database.

\begin{tabular}{|c|}
\hline $\begin{array}{l}\text { Input: } R ; \quad \text { Ouput: } R^{\prime} \text {. } \\
\text { for all } \mathrm{p} \in \mathrm{OK} \mathrm{K}_{\mathrm{B}-\mathrm{prices}} \mathrm{do} / / \text { cycle for the body } \\
\mathrm{i} \text {-list- } \mathrm{b}=\mathrm{i} \text {-list-b } \cup \sigma_{\text {price }}=p(\mathrm{FD}(\text { item,price })) ; \\
\text { for all } \mathrm{p} \in \mathrm{OK} \mathrm{K}_{\mathrm{H}-\mathrm{prices}} \mathrm{do} / / \text { cycle for the head } \\
\mathrm{i} \text {-list-h }=\mathrm{i} \text {-list-h } \cup \sigma_{\text {price }}=p(\mathrm{FD}(\text { item,price })) ; \\
\text { for all rules } r \text { in } R \text { do } \\
\text { if all items in r.body are in i-list-b and } \\
\quad \text { all items in r.head are in i-list-h then } \\
\quad \text { add } \mathrm{r} \text { to } R^{\prime} \text {; }\end{array}$ \\
\hline
\end{tabular}

Figure 2: The generation of $R^{\prime}$ from $R$ when functional dependencies are present.

\section{CONCLUSIONS}

This paper is a first step towards the construction of optimizers for a constraint based mining language. It provides the guidelines for the comparison of classes of queries by means of the existing relationships between their result sets. This is the premise to allow the reuse of the result sets of the queries when certain conditions are met. Furthermore in this paper we identify the database schema informations (unique constraints and functional dependencies) that can be exploited for these purposes. In these cases we show that the volume of the database to be processed by the mining engine is greatly reduced or completely avoided in a large subset of the cases. Furthermore, we identify a set of intermediate data structures called the mining indices specifically designed in order to exploit previous query executions. We show the practical implications of the discussed principles with a set of algorithms designed for a specific mining language, the MINE RULE operator.

Future work is to provide an implementation of the set of algorithms proposed here, taking into consideration the previous work on constraint-based mining and condensed representations. These ones, indeed, exploit the specific properties of each constraints (such as anti-monoticity and succinctness) in order to reduce at a minimum the global workload.

\section{ACKNOWLEDGMENTS}

This work has been funded by EU FET project cInQ.

\section{REFERENCES}

[1] E. Baralis and G. Psaila. Incremental refinement of mining queries. In Proc. of the 1st Int'l Conf. on Data Warehousing and Knowledge Discovery, pages 173-182, Firenze, Italy, 1999.

[2] M. Botta, R. Meo, and M.L. Sapino. Incremental execution of the mine rule operator. Technical Report RT 66/2002, Dipartimento di Informatica, Università di Torino, Italy, May 2002.

[3] V. Ng D. Cheung, J. Han and C.Y. Wong. Maintenance of discovered association rules in large databases: An incremental updating technique. In Proc. of 1996 Int'l Conf. on Data Engineering, New Orleans, Louisiana, USA, February 1996.

[4] B. Goethals and J. Van den Bussche. A priori versus a posteriori filtering of association rules. In Proc. of SIGMOD Workshop on Research Issues in Data Mining and Knowledge Discovery, 1999.

[5] T. Imielinski and H. Mannila. A database perspective on knowledge discovery. Communications of the ACM, 39(11):58-64, November 1996.

[6] B. Jeudy and J-F. Boulicaut. Constraint-based discovery and inductive queries: application to association rule mining. In Proc. of the Science Foundation Exploratory Workshop on Pattern Detection and Discovery in Data Mining, Imperial College, London, September 2002.

[7] B. Jeudy and J-F. Boulicaut. Optimization of association rule mining queries. Intelligent Data Analysis journal, 6(5):195-224, 2002.

[8] B. Jeudy and J-F. Boulicaut. Using condensed representations for interactive association rule mining. In Proc. of the 6th European Conf. on Principles and Practice of Knowledge Discovery in Databases, Helsinki, Finland, 2002.

[9] H. Mannila and H. Toivonen. Levelwise search and borders of theories in knowledge discovery. Data Mining and Knowledge Discovery, 1(3):241-258, November 1997.

[10] R. Meo, G. Psaila, and S. Ceri. A new sql-like operator for mining association rules. In Proc. of the 22nd VLDB Conference, Bombay, India, Sept. 1996.

[11] R. Meo, G. Psaila, and S. Ceri. An extension of SQL for mining association rules. Data Mining and Knowledge Discovery, 2(2):195-224, 1998.

[12] R. Ng, L.V.S. Lakshmanan, J. Han, and A. Pang. Exploratory mining and pruning optimizations of constrained association rules. In Proc. $A C M$ SIGMOD'98, pages 13-24, Seattle, WA, 1998.

[13] R. Ng, L.V.S. Lakshmanan, J. Han, and A. Pang. Optimization of constrained frequent set queries with 2-variable constraints. In Proc. ACM SIGMOD'99, pages 157-168, Philadelphia, PA, 1998.

[14] J. Pei and J. Han. Can we push more constraints into frequent pattern mining? In Proc. of the Int'l Conf. on KDD, Boston, MA, August 2000.

[15] J. Pei, J. Han, and L. V. S. Lakshmanan. Mining frequent itemsets with convertible constraints. In Proc. 2001 Int'l Conf. on Data Engineering, Heidelberg, Germany, April 2001.

[16] J. Wijsen and R. Meersman. On the complexity of mining quantitative association rules. Data Mining and Knowledge Discovery, 2(3):263-281, 1998. 